\title{
Honoré de Balzac, Le Père Goriot. Histoire parisienne
}

\section{Patrick Berthier}

\section{(2) OpenEdition}

\section{Journals}

\section{Édition électronique}

URL : http://journals.openedition.org/studifrancesi/3784

DOI : 10.4000/studifrancesi.3784

ISSN : 2427-5856

\section{Éditeur}

Rosenberg \& Sellier

\section{Édition imprimée}

Date de publication : 1 décembre 2012

Pagination : $587-588$

ISSN : 0039-2944

\section{Référence électronique}

Patrick Berthier, « Honoré de Balzac, Le Père Goriot. Histoire parisienne », Studi Francesi [En ligne], 168 (LVI | III) | 2012, mis en ligne le 30 novembre 2015, consulté le 07 mars 2021. URL : http:// journals.openedition.org/studifrancesi/3784; DOI : https://doi.org/10.4000/studifrancesi.3784

Ce document a été généré automatiquement le 7 mars 2021.

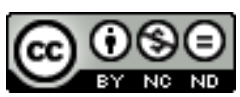

Studi Francesi è distribuita con Licenza Creative Commons Attribuzione - Non commerciale - Non opere derivate 4.0 Internazionale. 


\title{
Honoré de Balzac, Le Père Goriot. Histoire parisienne
}

\author{
Patrick Berthier
}

\section{RÉFÉRENCE}

HONORÉ DE BALZAC, Le Père Goriot. Histoire parisienne, édition critique enrichie d'un Cédérom, établie et présentée par Andrew OLIVER, Paris, Honoré Champion, 2011, pp. 312.

1 Dans quelques années cette édition sera un témoin intéressant de l'évolution de la recherche en la matière. Sa principale caractéristique est de ne pouvoir être utilisée sans le Cédérom encarté dans sa troisième de couverture. La partie "papier» de l'ouvrage ne donne en effet à lire, après une introduction de plus de soixante pages, que le texte nu du roman, sans appels de notes, mais hérissé de quelque 6500 appels de variantes: autant dire que ce n'est pas ici qu'on va relire Le Père Goriot pour son plaisir.

2 Avant d'ouvrir le disque, voyons l'introduction (seul élément de l'édition à n'être livré que sous la forme papier). Andrew Oliver y rappelle assez rapidement, parfois en reprenant des éléments de ses articles antérieurs, le contexte dans lequel le roman est né, sa rédaction, sa publication, la relation entre «imaginaire et écriture» (p.28), la complexe histoire de la page de titre, et des titres de parties dont le nombre a changé. La façon dont se présente le manuscrit, la manière dont l'écriture (matérielle) donne des indications sur le travail de l'écrivain, les changements apportés en cours de route et leurs implications (Balzac avait d'abord placé l'action en 1824), les sources littéraires donnent lieu à des développements qui recoupent, et c'est naturel, ce que les éditions critiques précédentes avaient dit, tout en apportant des remarques nouvelles permises, notamment, par la minutie avec laquelle l'examen du manuscrit et des variantes a été mené; il est dommage, quand A. Oliver se trouve en désaccord avec ses prédécesseurs, s'agît-il de Pierre-Georges Castex ou de Stéphane Vachon, qui ont tout de même fait un peu plus que déblayer le terrain, qu'il le dise souvent sur un ton si péremptoire. 
On arrive au point le plus intéressant de cette introduction lorsque A. Oliver parle des éditions du roman; le rappel précis qu'il en fait l'amène à justifier (pp. 63-69) le choix qu'il a fait d'adopter pour la sienne non pas, suivant la tradition, le texte le plus tardif (que nous appelons le «Furne corrigé», parce qu'il reprend les corrections de Balzac sur son exemplaire de La Comédie humaine), mais celui de l'édition originale de 1835. Les raisons avancées sont puisées aux «principes de la textologie moderne», qui «refus[e] la notion traditionnelle de "texte définitif"» (p. 64). A. Oliver estime que l'on respecte mieux la nature de ce que fut Le Père Goriot à sa publication en donnant le texte de l'originale, fortement modifié ensuite pour ce qui concerne le vocabulaire, la ponctuation, et même la proximité du romancier avec ses propres créatures. L'exposé d'A. Oliver est passionnant, notamment la longue note de la p. 69 où il observe (après d'autres) qu'aucun lecteur du temps de Balzac n'a pu lire le «Furne corrigé». Il a beau jeu, pour prévenir le désaccord des balzaciens traditionalistes, de leur dire qu'ils peuvent reconstituer cet état définitif (pour lui sans signification) puisque le disque joint au volume comporte toutes les variantes du texte. C'est évidemment le «bonus» le plus visible de son travail, les éditions critiques antérieures et notamment celle, pionnière, de P.-G. Castex (Garnier, 1960), n'ayant pu en donner qu'une sélection.

Lorsqu'on ouvre le Cédérom, il faut aller tout de suite à la table pour éviter divers flottements dans les possibilités de circuler à l'intérieur d'un dossier très buissonnant. Le «Protocole», assez complexe, explique comment naviguer dans le disque, et la manière dont sont transcrites les variantes. Le texte du roman (pp. 91-300) peut être atteint page par page, en cliquant sur le numéro de la page dans la table. Le texte s'affiche sur la moitié gauche de l'écran, et quand on clique sur un appel de variante, celle-ci s'affiche sur la moitié droite. En outre, un certain nombre de noms propres et de mots sont surlignés: c'est l'équivalent d'appels de notes, invisibles à la lecture de la version papier puisqu'elle est imprimée en noir et blanc. Cliquer sur ces noms ou ces mots permet soit de se remémorer les autres romans dans lesquels apparait tel ou tel personnage, soit de lire les définitions des mots rares ou datés, tirées des dictionnaires courants (le Larousse du XIX siècle, le Littré, le Trésor de la langue française), soit encore d'accéder à des pages internet diverses, précieuses quand elles proposent une iconographie, parfois connue (les illustrations du Furne), parfois moins (les lieux du roman en images et gravures d'époque). Le disque comporte encore un dossier de presse du roman et de ses adaptations théâtrales de 1835, et une bibliographie étendue.

5 Le travail accompli mérite le respect. On regrette qu'il n'ait pas bénéficié d'une relecture ultime, qui aurait permis d'en éliminer quelques scories, les unes matérielles (p. 46 de l'introduction, une note est restée vide), les autres de contenu: le titre du périodique dans lequel le roman a été prépublié est «Revue de Paris», sans article, comme l'indiquent d'ailleurs correctement les notes, alors que dans le texte de l'introduction - c'est visible p. 52, par exemple - on a imprimé systématiquement «La Revue de Paris» (qui est une revue de la fin du XIX ${ }^{e}$ siècle). La maîtrise de la langue française est à peu près parfaite, à des broutilles près, mais que l'on trouve sans cesse sous la plume des scripteurs francophones («compte rendu» avec un trait d'union, par exemple). J'ai dit que la lecture du volume imprimé est gênée par le nombre des appels (plus de cinquante dans la même page pour les passages les plus corrigés); elle l'est aussi par une gestion bizarre de la typographie: de façon aléatoire, des passages entiers sont imprimés en caractères élargis d'un point, ce qui inflige à l'œil une étrange épreuve: ce phénomène n'apparaît pas si on lit le texte dans la version électronique (on 
peut faire le test de la différence sur les pp. 148 ou 286, par exemple: c'est spectaculaire). La disposition typographique elle-même souffre de défauts: des ponctuations en tête de ligne faute d'espaces insécables (par exemple p. 107, ou p. 175 un «jusqu/'au» qui fait sursauter), ou un alinéa fautif p. 120 («la dernière lettre de sa / femme»): ces défauts n'existent pas sur le disque, seulement sur le tirage papier, sans doute à la suite de manipulations chez l'éditeur. Enfin une faute au moins, dans le texte de Balzac cette fois, est vraiment regrettable: au lieu du joli «chanteronna» qui qualifie la désinvolture de Maxime de Trailles («cet impertinent [...] chanteronna quelque roulade italienne»), la version papier et la version électronique donnent: "chantonnera», qui n'a bien sûr aucun sens. Il suffit de cliquer sur les symboles qui permettent de lire le même passage dans les différentes éditions, pour voir que cette faute n'y figure pas, et notamment pas chez Werdet (voir p. 154 de cette édition, et p. 130 de sa transcription). Cette erreur ne compromet pas le statut de chef-d'œuvre du Père Goriot, mais quand on écrit: «Castex a tort» (p. 44 de l'introduction, n. 2), on doit soi-même être irréprochable.

Il serait inélégant de s'en tenir à cette pique, quelque tentation qu'on en ait, tant il est évident que ce Père Goriot marque une date. Comme toute première version d'un outil nouveau, celle-ci sera améliorée par les éditeurs d'autres romans. Mais tant qu'à utiliser l'électronique, il aurait été plus radical et plus satisfaisant de mettre en vente purement et simplement un Cédérom, en y incluant évidemment l'introduction qui en est ici absente: car en réalité on n'a pas besoin du volume, puisque le disque contient tout, et se révèle d'un maniement assez facile. L'avenir est à la véritable édition critique intégrale électronique, et seulement électronique: Andrew Oliver a montré la route à suivre. Pour ce qui est de relire Le Père Goriot seulement pour le bonheur de le relire, chacun restera fidèle à telle vieille édition dans laquelle il l'a découvert jadis, et qu'il feuillettera, comme le dit l'auteur lui-même (ici p. 92), «dans un moelleux fauteuil». 